\title{
Is there a role for stenting in case of acute esophageal variceal bleeding?
}

Author

Institution
Gilles Lesur

Department of Gastroenterology, Ambroise Paré Hospital, Boulogne, France submitted 29. July 2014 accepted after revision 27. August 2014

\section{Bibliography}

Dol http://dx.doi.org/ 10.1055/s-0034-1390758

Published online: 29.10.2014

Endoscopy International Open 2014; 02: E197-E198

(c) Georg Thieme Verlag KG Stuttgart . New York E-ISSN 2196-9736

\section{Corresponding author}

\section{Gilles Lesur, MD}

Department of

Gastroenterology

Ambroise Paré Hospital

9 avenue Charles de Gaulle

92100 Boulogne

France

Fax: +33149095880

gilles.lesur@apr.aphp.fr
Pharmacological therapy with endoscopic band ligation (EBL) fails in 10 to $20 \%$ of patients to control acute bleeding caused by rupture of esophageal varices [1]. Initial failure to control bleeding or early rebleeding has a significant impact on mortality. To reduce the incidence of treatment failure and death, more aggressive therapies may be used in patients with high risk of treatment failure. Rescue therapy is indicated when endoscopic treatment combined with pharmacological therapy has failed to control bleeding. In this situation, there are three possibilities: (1) the balloon tamponade (BT); (2) the insertion of a transjugular intrahepatic portosystemic shunt (TIPS); and (3) the insertion of an esophageal covered self-expanding metal stent (SEMS).

The conclusions of the Baveno $V$ consensus workshop published in 2010 concerning management of treatment failures are: (1) BT should only be used in massive bleeding as a temporary "bridge" until definitive treatment can be instituted (for a maximum of $24 \mathrm{~h}$, preferably in an intensive care facility); (2) persistent bleeding despite combined pharmacological and endoscopic therapy is best managed by TIPS with covered stents; (3) rebleeding during the first 5 days may be managed by a second attempt at endoscopic therapy; however, if rebleeding is severe, TIPS with covered stents is likely to be the best option; and (4) uncontrolled data suggest that SEMS may be an option in refractory esophageal bleeding, although further evaluation is needed [2].

Since this workshop, there have been no new data concerning BT, an old procedure with several disadvantages: need of skilled personnel, high incidence of serious complications, success rate in achieving short-term hemostasis between 50 to $90 \%$, frequent rebleeding on removal of the tube [3]. Concerning this point, nothing has changed and $\mathrm{BT}$ remains only a holding measure until a definitive procedure can be performed.
Conversely, interesting data concerning SEMS in this emergency context have been published. In this issue of EIO, Kinesh et al. publish an exhaustive review evaluating the technical feasibility, efficacy, and safety of SEMS as a rescue for acute variceal bleeding among 108 patients (91 males, 17 females). Mean age calculated from all reported cases was 54.3, as expected for cirrhotic patients. Of the patients 48 were reported to have Child-Pugh score class C and 32 were class B. In most studies collected by Kinesh et al., SEMS is a nitinol, removable, covered-by-polyurethanefoil, self-expanding metal stent. The stent placement (with a guidewire) and the removal (with retrieval loops) could be easily done endoscopically without radiological guidance, making it a more practical therapeutic intervention to stabilize a bleeding patient. SEMS was successfully deployed in 100 out of 108 cases; immediate hemostasis was obtained among 96 patients, and rebleeding occurred with only 4 patients. Stents remain safely intact for 4 to 14 days in most cases. Successful SEMS extraction was performed in almost all cases under endoscopic guidance without any reported technical difficulty. Stent migration, which was the most common complication, was observed in $21 \%$ of the patients but not associated with rebleeding. Unfortunately, a total of 27 patients ( $25 \%$ ) died within 7 to 60 days, mostly due to progressive hepatic decompensation and/ or multiorgan failure.

These results must be compared with those of TIPS. Acute variceal bleeding that is poorly controlled with endoscopic therapy is generally well controlled with TIPS, which has a success rate of 90 to $100 \%$. However, TIPS has a mortality rate of 27 to $50 \%$ [4]. As with SEMS, death occurring within 30 days of the procedure is most commonly caused by multiorgan failure, and death more than 30 days following the procedure is most commonly related to liver failure. Increased mortality is related to a Child-Pugh class C clinical sta- 
tus, hemodynamic instability at the time of the TIPS procedure, and the presence of comorbidities. Many studies reporting on emergency TIPS for the rescue treatment of acute esophageal varices bleeding have shown low survival rates and significantly higher mortality in comparison with patients undergoing elective TIPS [4]. In one study, 42 out of 123 patients (34.1\%) died within 30 days of TIPS for acute bleeding, whereas only $16.5 \%$ died following elective TIPS creation [5].

Patients with a Child-Pugh class A score and whose bleeding does not appear life-threatening should probably first be managed by further sessions of endoscopic treatment. Conversely, patients with more advanced liver disease who have had a single massive bleed and unsuccessful endoscopic treatment on one occasion, may be better treated by SEMS or TIPS rather than undergoing a second endoscopic therapy session. For patients with Child-Pugh class $\mathrm{C}$ or class $\mathrm{B}$ disease with active bleeding who are admitted for acute variceal bleeding, García-Pagán et al. [6] have demonstrated that early TIPS with a covered stent is associated with significant reduction in the failure to control bleeding, in rebleeding, and in mortality with no increase of hepatic encephalopathy. This study clearly favors an early use of TIPS in the context of persistent variceal bleeding. This benefit is probably related to reducing the duration or risk of hypotension that is likely to be detrimental for patients with decompensated liver disease. There are no data concerning the early use of SEMS but such a benefit could also be expected with this method.

In conclusion, SEMS is a safe and effective treatment with relatively few adverse outcomes for rescue therapy for refractory acute variceal bleeding. TIPS is also another highly effective op- tion for hemostasis. Emergency SEMS or TIPS should be considered early in patients with refractory esophageal variceal bleeding before the clinical condition worsens, to prevent severe hepatic dysfunction. Final decisions should be based on the individual patient, overall clinical conditions, and local availabilities and expertise, ideally in specialized centers. In the future, the two modern options - that is, SEMS and TIPS - must be compared in a randomized trial for a more scientific approach to this difficult but not infrequent situation.

\section{Competing interests: None}

\section{References}

1 Garcia-Tsao G, Bosch J. Management of varices and variceal hemorrhage in cirrhosis. N Engl J Med 2010; 362: 823-832

2 De Franchis $R$, on behalf of the Baveno $\mathrm{V}$ faculty Revising consensus in portal hypertension: report of the Baveno $\mathrm{V}$ consensus workshop on methodology of diagnosis and therapy in portal hypertension. J Hepatology 2010; 53: $762-768$

3 Panés J, Terés J, Bosch J et al. Efficacy of balloon tamponade in treatment of bleeding gastric and oesophageal varices. Results in 151 consecutive patients. Dig Dis Sci 1988; 33: 454-459

4 Loffroy R, Estivalet L, Cherbalnc V et al. Transjugular intrahepatic portosystemic shunt for the management of acute variceal bleeding. World J Gastroenterol 2013; 19: 6131 -6143

5 Rajan DK, Haskia ZJ, Clark TW. Serum bilirubin and early mortality after transjugular intrahepatic portosystemic shunts: results of a multivariate analysis. J Vasc Interv Radiol 2002; 13: 155-161

6 García-Pagán JC, Caca K, Bureau C et al. Early use of TIPS in patients with cirrhosis and variceal bleeding. N Engl J Med 2010; 362: 2370 2379 